\title{
Langmuir
}

pubs.acs.org/Langmuir

(C) 2009 American Chemical Society

\section{Engineering Transfer of Micro- and Nanometer-Scale Features by Surface Energy Modification}

\author{
Barbara Cortese, ${ }^{* \dagger \dagger}$ Claudia Piliego $,{ }^{*}, \dagger$ Ilenia Viola, Stefania D’Amone, Roberto Cingolani, and \\ Giuseppe Gigli
}

National Nanotechnology Laboratories (NNL) of CNR-INFM, Distretto Tecnologico, Università del Salento, Via Arnesano 16, Lecce 73100, Italy. ${ }^{\dagger}$ Both authors have contributed equally in the preparation of this work.

Received January 20, 2009. Revised Manuscript Received April 3, 2009

\begin{abstract}
Micropatterning of surfaces is gaining importance in various applications ranging from biosensors to microfluidic and lab-on-a-chip devices, where the control of the surface chemistry is of great importance for the application. In this paper, we introduce a patterning technique of topographical features, which is applicable on different substrates by modifying their surface energy. The textured surface is obtained via polydimethylsiloxane (PDMS) transfer, and the topographical parameters can be systematically tailored by selective treatment with oxygen plasma of either the PDMS stamp, the substrate, or both. Our approach is an alternative technique to create micro- and nanopatterns of various height and shape over a large area on different substrates. The possibility to control cell behavior on different surfaces tailored with this microtransfer patterning approach was also evaluated. The cell culture on patterned surfaces showed the possibility of modulating cell adhesion. Our method is based on simple transfer of silicone elastomeric patterns to the surface, and therefore, it is very simple and fast compared to other complex techniques. These observations could have implications for tissue-scaffold engineering science in areas such as microfluidic devices and control of cell adhesion.
\end{abstract}

\section{Introduction}

Surfaces of controlled topography are of particular importance for many technological and biological applications because they influence cell behavior in terms of adhesion and differentiation ${ }^{1-3}$ and provide mechanical cues within tissue-scaffold engineering, microfluidic devices, ${ }^{4}$ and drug delivery. ${ }^{5}$ In fact, the control of features at length scales ranging down from micro- to nanometers $^{6}$ allows for an exceptional ability to influence the cellular microenvironment as, for example, by reproducing the architecture and geometry of tissues, which would allow for the manipulation of cell-cell interactions or the control of the shape of cells. ${ }^{7}$ Soft lithography has drawn consistently attention in microand nanopatterning surfaces as an alternative to photolithographic techniques, because of its aptitude for patterning large areas and a wide assortment of substrates using simple and low-cost techniques with high throughput..$^{8-10}$ In fact, it consists of a complementary array of techniques based on pattern transfer using an elastomer, such as polydimethylsiloxane (PDMS). ${ }^{6}$

Up to now, many methods have been proposed to pattern on specific substrates, such as photochemical methods, ${ }^{11}$

*To whom correspondence should be addressed. E-mail: barbara.cortese $@$ unile.it (B.C.); claudia.piliego@unile.it (C.P.).

(1) Singhvi, R.; Stephanopoulos, G.; Wang, D. Biotechnol. Bioeng. 1994, 43, 764-771.

(2) Curtis, A.; Wilkinson, C. Biomaterials 1997, 18, 1573-1583

(3) Dalby, M.; Riehle, M.; Yarwood, S.; Wilkinson, C.; Curtis, A. Exp. Cell Res. 2003, 284, 274-282.

(4) Sia, S.; Whitesides, G. Electrophoresis 2003, 24, 3563-3576.

(5) Bhushan, B.; Burton, Z. Nanotechnology 2005, 16, 467-478.

(6) Whitesides, G. M.; Ostuni, E.; Takayama, S.; Jiang, X.; Ingber, D. E. Annu. Rev. Biomed. Eng. 2001, 3, 335-373.

(7) Chen, C. S.; Mrksich, M.; Huang, S.; Whitesides, G. M.; Ingber, D. E. Science 1997, 276, 1425-1428.

(8) Zankovych, S.; Hoffmann, T.; Seekamp, J.; Bruch, J. U.; Sotomayor Torres,

C. M. Nanotechnology 2001, 12,91.

(9) Chou, S. Y.; Krauss, P. R.; Renstrom, P. J. Science 1996, 272, 85

(10) Li, M.; Wang, J.; Zhuang, S. Y.; Chou, S. Y. Appl. Phys. Lett. 2000, 76, 673.

(11) Blawas, A. S.; Reichert, W. M. Biomaterials 1998, 19, 595-609.

(12) Kane, R. S.; Takayama, S.; Ostuni, E.; Ingber, D. E.; Whitesides, G. M. Biomaterials 1999, 20, 2363-2376. microcontact printing, ${ }^{12,13}$ colloidal assembling, ${ }^{14}$ microfluidic systems, ${ }^{12,15}$ and spraying deposition methods, such as inkjet pattering. ${ }^{16}$ Recently, among the large variety of methods, a new soft lithographic method for micropatterning substrates, known as decal transfer microlithography (DTM), ${ }^{17}$ has been developed to transfer elastomeric decals, by means of cohesive mechanical failure (CMF), exploiting the interfacial bonding of PDMS on different substrates and subsequent rupture of the PDMS to obtain the pattern. This technique proved to be very useful for patterning micrometer-sized PDMS patterns on flat or curved large areas. ${ }^{18}$ However, conventional DTM involves strict conditions, because the adhesion required is induced by the use of a $\mathrm{UV} /$ ozone (UVO) pretreatment of PDMS. Recent reports have described variations in the activation and transfer step of this technique. Ruoff and co-workers have described an alternative procedure called bond-detach lithography, ${ }^{19}$ where patterning is achieved by bonding and detaching a PDMS mold from a PDMS film and rupturing the film in correspondence of the patterns. Another novel technique has been described by Huck et al., ${ }^{20}$ where bonding is based on the formation of silicate species because of the reaction between $\mathrm{NaOH}$ and the PDMS stamp after hydrolyzing the stamp. Although the use of PDMS to pattern by conformal contact has been reported, to the authors' knowledge, a control of the patterned features has not yet been obtained. This aspect is particularly important because it could

(13) Bernard, A.; Renault, J. P.; Michel, B.; Bosshard, H. R; Delamarche, E. Adv. Mater. 2000, 12, 1067-1070.

(14) Yapb, F. L.; Zhang, Y. Biomaterials 2007, 28, 2328-2338.

(15) Delamarche, E.; Bernard, A.; Schmid, H.; Michel, B.; Biebuyck, H. Science 1997, 276, 779-781.

(16) Morozov, V. N.; Morozova, T. Y. Anal. Chem. 1999, 71, 3110-3117

(17) Childs, W. R.; Nuzzo, R. G. J. Am. Chem. Soc. 2002, 124, 13583-13596.

(18) Ahn, H.; Shim, A.; Lee, K. J.; Childs, W. R.; Rogers, J. A.; Nuzzo, R. G. J. Appl. Phys. 2006, 100, 084907-7.

(19) Thangawng, A. L.; Swartz, M. A.; Glucksberg, M. R.; Ruoff, R. S. Small 2007, 3, 132-138.

(20) Zheng, Z.; Azzaroni, O.; Zhou, F.; Huck, W. T. S. J. Am. Chem. Soc. 2006, $128,7730-7731$. 
offer several advantages and open the way to new applications. Our method differs in the use of an oxygen plasma treatment, improving the results obtained with traditional techniques without the use of corrosive substances and by simplifying the procedure steps. Addressing the intrinsic constraints brought up by these techniques, we alternatively propose a single-step procedure to pattern different substrates by basic transfer of PDMS through simple conformal contact.

Therefore, the present work covers the issue of intentional and controllable transfer of PDMS patterning. In this context, activation of the surface was obtained by means of an oxygen plasma treatment, increasing surface energy and improving adhesion for transfer. Specifically, the local control of the height of the transferred PDMS by simply alternating the treatment of surfaces and PDMS stamp allowed us to obtain features ranging from micro- to nanometric scale and complex patterns with multiple printing processes.

\section{Experimental Approach}

Preparation of SU-8 Masters and Substrates. Patterns were prepared using SU-8 2010, purchased from MicroChem Coorporation, on silicon wafer substrates. The patterning was carried out using the Karl Suss MJ B3 mask aligner with UV illumination and a photomask containing the patterns. Different substrates used for transfer, such as microscope glass slides and indium-tin-oxide (ITO)-coated slides, were ultrasonically cleaned in acetone and isopropanol for $10 \mathrm{~min}$, respectively, and subsequently dried with a $\mathrm{N}_{2}$ air flow before use.

Preparation of PDMS Samples. PDMS (Sylgard 184), an elastomeric material composed of an elastomer base and a curing agent, was purchased from Dow Corning (Midland, MI). Samples were generally prepared from a 10-20:1 mixture (by weight) of prepolymer and curing agent, poured onto the masters, and left to settle for $10 \mathrm{~min}$, so that the trapped air bubbles could emerge to the surface. After the removal of all of the air bubbles, the mixture was put into an oven $\left(80^{\circ} \mathrm{C}\right)$ for curing for $2 \mathrm{~h}$. After curing, the PDMS sample was gently peeled from the mold.

Plasma Treatment. The microstructured PDMS samples were exposed to an oxygen plasma generated in a RIE IONVAC inductively coupled (ICP) plasma reactor (PGF 600 RF HUTTER; plasma conditions: $200 \mathrm{~W}, 40 \mathrm{mTorr}$ ) for $30 \mathrm{~s}$.

Characterization. The samples were characterized using scanning electron microscopy (SEM) and atomic force microscopy (AFM). For electron microscopy, the microstructure was observed and evaluated using a LEICA STEREOSCAN 440 SEM operating at $20 \mathrm{kV}$

AFM measurements were carried out in air, in tapping mode, using a SMENA MT-DTA atomic force microscope, to analyze the transferred features on the structure. Each reported height measurement was calculated on the average of 10 individual experiments. The height and size of transferred features fabricated from identical patterned stamps was reproducible to a standard error within $10 \%$ of the mean values obtained.

Cell Culture. Infinity telomerase immortalized human fibroblasts (hTERT-BJ1, Clonetech Laboratories, Inc., Palo Alto, CA) were grown in Dulbecco's modified Eagle's medium (DMEM) supplemented with $10 \%$ fetal bovine serum (FBS). Cells were cultured at $37{ }^{\circ} \mathrm{C}$ under humidified $10 \% \mathrm{CO}_{2}$. Seeding densities were $10^{4}$ cells $/ 6$ well cluster dish. About $2 \mathrm{~mL}$ of a $10^{5}$ cells $/ \mathrm{mL}$ suspension was seeded onto patterned substrates. Cells were fixed within $4 \%$ formaldehyde/PBS, with $1 \%$ sucrose at $37{ }^{\circ} \mathrm{C}$ for 15 min. After cells were permeabilized with 5\% Triton X-100 (Sigma) in PBS and blocked with 1\% BSA (Sigma) in PBS (Invitrogen), they were incubated in mouse antibody vinculin (Sigma) and phalloidin (Intritrogen) at $37^{\circ} \mathrm{C}$, following by $1 \mathrm{~h}$ of incubation with biotinynalated anti-mouse $\operatorname{IgG}(\mathrm{H}+\mathrm{L})$ secondary antibody (Vector Laboratories). Subsequently, cells were stained with a third antibody Texas Red STREPTAVIDIN (Vector Laboratories). Each antibody was diluted at 50:1, in a BSA solution in PBS. The samples were rinsed 3 times for $5 \mathrm{~min}$ in $0.5 \%$ Tween 20/PBS between each staining. The surfaces were mounted with Vectashield DAPI (Vector Laboratories) and then viewed by a fluorescence microscope (Zeiss Axiovert 200M).

\section{Results and Discussion}

Patterning of surfaces through modification of the surface energy by placing a PDMS stamp on a substrate via conformal contact provides a new method for achieving feature transfer.

Elastomers based on PDMS are renowned to be composed of a prepolymer and a cross-linker, consisting of repeating silicon hydride $-\mathrm{OSiHCH}_{3}-$ units with a high mobility of the polymer chains. There is, in fact, a high diffusion of the oligomers from the bulk of the stamp to the surface, ${ }^{21,22}$ causing dynamic changes on the surface of the PDMS stamp. Therefore, transfer of lowmolecular-weight residues of PDMS from the stamp onto the surface takes place once the stamp is placed in conformal contact on the substrate after a certain amount of time. The amount of molecules transferred is of the order of nanometric features caused by the rupture of the weak bonds formed between the stamp and the substrate. After the removal of the PDMS stamp, which can be reused, residues of the pattern are present on the substrate. Activation of the surface by oxidation processes increases the strength of bonding as the concentration of hydroxyl groups increases. This leads to the formation of stronger intermolecular bonds ${ }^{23,24}$ and, as a consequence, to an enhanced amount of transferred PDMS. Ye et al. have shown that techniques used for oxidation of PDMS, such as UV, UVO, and exposure to oxygen plasma (OP), developed silanol groups $(-\mathrm{OH})$ at the expense of methyl groups. The comparison among these processes indicated differences in the response of molecular structural and orientation changes at the PDMS-air interface. ${ }^{25}$ They also showed that a major presence of $-\mathrm{OH}$ groups with a reduction of $\mathrm{CH}_{3}$ groups on a PDMS surface was more rapid for OP exposure than for UVO and UV treatments. Moreover, OP modification quickly produced hydroxyl-terminated groups on the PDMS surface, replacing the $\mathrm{CH}_{3}$ groups, while a fraction of residual methyl groups $\mathrm{CH}_{3}$ was left with $\mathrm{UV}$, along with UVO modification. Thus, OP treatment resulted to be more rapid and efficient at the PDMS interface, for establishing a stronger and highly stable bonding of the PDMS monomers to the surface. In the studies carried out here, we show the possibility to tailor the transferred features ranging from nano- to micrometer size as a function of the treatment of the surfaces.

A schematic illustration of the PDMS transfer steps is reported in Figure 1. Geometrical patterns of various shapes and sizes were fabricated by simply casting different ratios of PDMS 10:1/ 20:1 (Sylgard 184 silicone elastomer kit, Dow Corning, Midland, MI) onto a master in SU8 bearing a relief pattern. After the PDMS was cured for at least $2 \mathrm{~h}$ at $80^{\circ} \mathrm{C}$, the stamps were peeled off from the masters. Briefly, the elastomeric poly(dimethylsiloxane) (PDMS) mold was used as a master to transfer patterns of PDMS.

(21) Glasmästar, K.; Gold, J.; Andersson, A. S.; Sutherland, D. S.; Kasemo, B. Langmuir 2003, 19, 5475-5483.

(22) Wang, X. J.; Östblom, M.; Johansson, T.; Inganäs, O. Thin Solid Films 2004, 449, 125-132.

(23) Hillborg, H.; Gedde, U. W. Polymer 1998, 39, 1991-1998.

(24) Chabinyc, M. L.; Chiu, D. T.; McDonald, J. C.; Stroock, A. D.; Christian,

J. F.; Karger, A. F.; Whitesides, G. M. Anal. Chem. 2001, 73, 4491-4498.

(25) Ye, H.; Gu, Z.; Gracias, D. H. Langmuir 2006, 22, 1863-1868. 

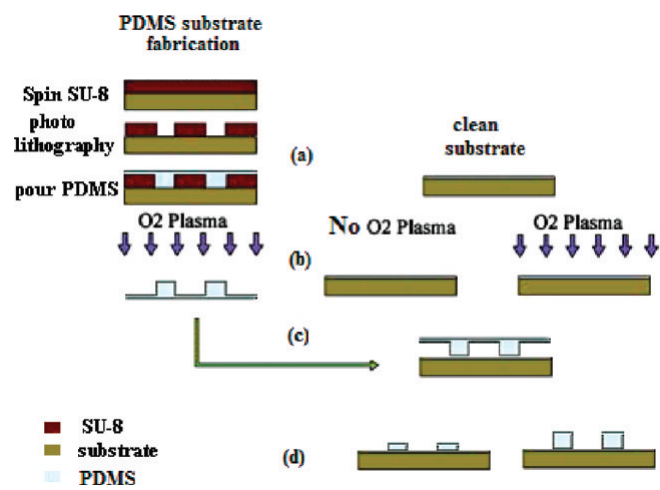

Figure 1. Schematic illustration of the steps involved in the PDMS transfer patterning: (a) PDMS stamp with the desired topography casted and cured on the masters, (b) PDMS stamp treated with or without oxygen plasma, (c) PDMS stamp pressed on the substrate, adequately treated, and (d) stamp removed to reveal the pattern.

As previously indicated, the key element of this procedure is the conformal contact between the elastomer stamp and the substrate. Note that the degree of transfer of the PDMS stamp was tuned by controlling the contact time and oxygen plasma treatment of the surfaces. Nanometric features, $\sim 10-100 \mathrm{~nm}$, were transferred when both the patterned PDMS stamp and the substrate were not treated. When the patterned PDMS stamp, substrate, or both were treated with oxygen plasma (30 s), the height of the transferred features increased up to the micrometer scale. After exposure, the PDMS stamp was immediately placed in conformal contact with the appropriately modified substrate, by carefully lowering the patterned stamp to the substrate until the two surfaces formed a complete contact. Once bonded to a surface, the contact time varied from 1 to $72 \mathrm{~h}$, depending upon the treatment and the desired height, and while maintaining contact between the stamp and substrate, they were placed in an oven at $60^{\circ} \mathrm{C}$. Subsequently, the PDMS stamp was peeled off to reveal patterns transferred to the surface. The higher amount of transferred PDMS features is due to an irreversible sealing between the PDMS and the substrates. Oxygen plasma leads indeed to the formation of covalent bonds present on both stamp and substrate, which increases the strength of the interfacial bonds. ${ }^{26}$ The peeling of the PDMS, consequently, causes the rupture of the bonds formed between the $-\mathrm{OH}$ radicals on the surface of the PDMS stamp and the substrate. Through the modulation of experimental conditions, the dimensions of the transferred features were easily tailored. According to the height desired, the substrate was treated or not, as shown in Figure 2. Pressure was not applied on the stamp to avoid an undesirable larger contact area, which would result in a transfer of larger features. The PDMS stamps used consisted of lines and squares. The pattern shown in Figure 2 was made by transferring PDMS lines to a planar ITO or glass substrates. The structure transferred shown in Figure 2a was produced by treating both the PDMS stamp and the substrate with oxygen plasma, while in Figure 2b, only the PDMS stamp was plasmatreated. The patterns differ in height and quantity of the transferred PDMS because of the different treatment of the substrates.

Consequently, no plasma treatment of the substrate induced a transfer of features only along the edges of the pattern. The

(26) McDonald, J. C.; Duffy, D. C ; Anderson, J. R.; Chiu, D. T.; Wu, H Schueller, O. J. A.; Whitesides, G. M. Electrophoresis 2000, 21, 27-40. surface tension of the stamp, because of the hydrophobicity of the substrate, did not allow for a conformal contact of the whole area. A more detailed analysis was made with AFM scans. Typical AFM images of the transferred PDMS patterns deposited by conformal contact, where only the PDMS stamp was treated with oxygen plasma, are shown in Figure 3.

In this regime, it is possible to downscale the size of the transferred features. The choice of the treatment of the surfaces was crucial for the efficacy of the method. In fact, nanopatterns with high-quality defined topographical features were achieved when neither the stamp nor the substrate was treated, as shown in the AFM images in Figure 4. We ascribed this different behavior to the relative high motility of the polymer chains from the bulk to the surface of the PDMS stamp and, subsequently, to the mechanical rupture of the bonds of the PDMS stamp. It was observed that the bonding between the PDMS stamp and the substrate was stronger if both PDMS and substrate were treated by plasma, increasing the polar interactions or the covalent bonds formed by the reactions of polar functionality. Therefore, when no treatment was performed, the amount of transferred PDMS was much less, resulting in a nanometric scale of the features. Figure 4 shows the 3D AFM image of the transferred nanoscale PDMS patterns with a cross-sectional analysis. The height of the transferred PDMS lines was $\sim 11 \pm 3 \mathrm{~nm}$.

An advantage in not treating the surfaces is that the same stamp can be repeatedly used in transferring the patterns, in contrast to the previous methods used for PDMS transfer. Note that we were able to control the transfer and to obtain patterning with nanoscale features.

To obtain these results, a systematic study regarding the mixing ratio of the PDMS/curing agent, the contact time, and the heating treatment was carried out. These factors contributed to affect the quality of the patterning features.

The mixing ratio of the PDMS/curing agent was found to have some influence on the degree of the transferred PDMS. A different ratio of PDMS base and curing agent was used to investigate the influence of the curing agent on the amount of transfer, because the physical and chemical characteristics change with the ratio and properties of these components. The commonly used 10:1 (m/m) ratio of PDMS monomer base to curing agent was found to yield the transfer of the border of the patterns. A higher concentration of curing agent leads to a larger number of linking chains, which restricts the physical expansion of the polymer network, ${ }^{27}$ leading the PDMS to be more rigid, therefore resulting in a lower degree of PDMS transfer. The transferred pattern with a 10:1 (m/m) ratio resulted in more uniform and accurate features ranging from $\sim 10 \mathrm{~nm}$ to $3.5 \pm 0.3 \mu \mathrm{m}$. While the concentration of the curing agent decreases, the topographical height increases, with higher features transferred, obviously because of the higher elasticity, but with a less uniform surface. We believe that the gaps between polymer chains caused by a lower mixing ratio would allow for a higher motility of the polymer chains from the bulk to the surface, leading to a minor strength. Thus, when both the stamp and substrate were treated with the oxygen plasma, higher features were achieved. Figure 5 shows a series of SEM images of a 20:1 ratio of PDMS patterns transferred onto glass and ITO slides after plasma treatment of both stamp and substrates, showing the transfer of both negative and positive patterns.

Figure 6 shows in major detail the lack of uniformity along the walls and on the top of the transferred features using a 20:1

(27) Flory, P. J. In Principles of Polymer Chemistry; Cornell University Press: Ithaca, NY, 1953 

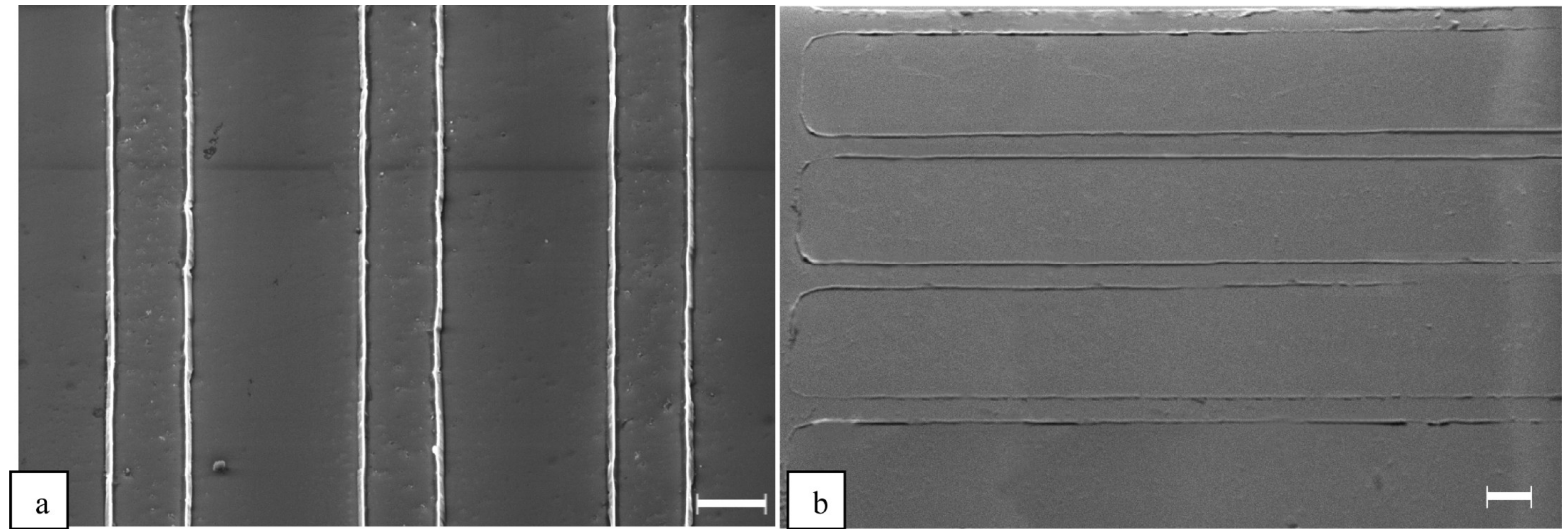

Figure 2. SEM image of PDMS lines transferred on (a) a substrate plasma treated after $1 \mathrm{~h}$ of contact time and (b) a substrate without plasma treatment after $24 \mathrm{~h}$. Both PDMS stamps were oxidized with a plasma treatment and were placed in an oven at $60{ }^{\circ} \mathrm{C}$ for the time of contact. The amount of PDMS transferred on the treated surface is much higher than the quantity on the untreated one. Scale bars are $40 \mu \mathrm{m}$.
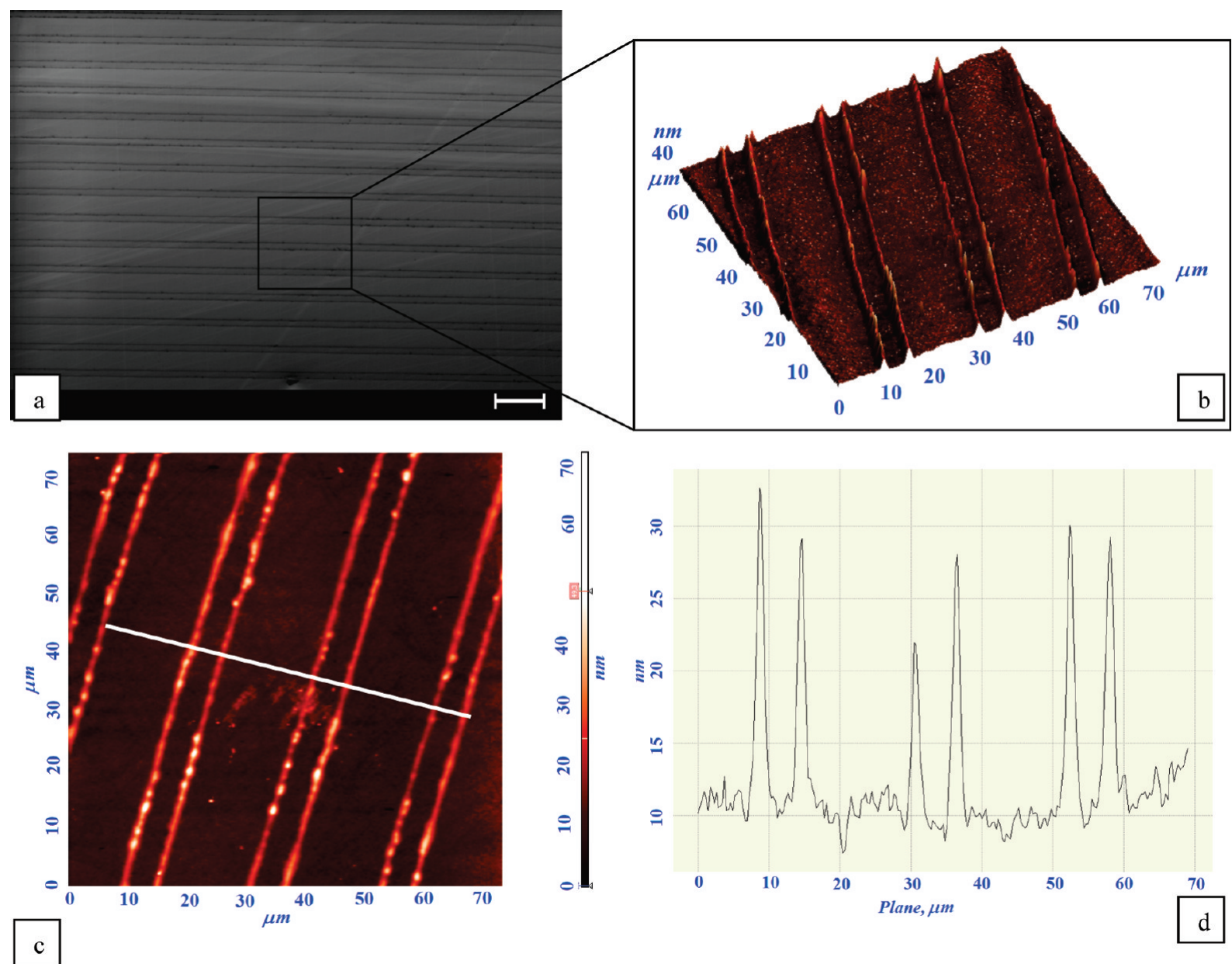

Figure 3. (a) SEM and (b-d) AFM scans of the patterned substrates with a PDMS stamp having lines. The scale bar is $30 \mu \mathrm{m}$. Only the PDMS stamps were plasma-treated. The contact time between the PDMS stamp and surfaces was $1 \mathrm{~h}$. (b) 3D image of the AFM scan. (c and d) Cross-sectional profile of the AFM images.

PDMS ratio, thus because of the enhanced elasticity of the patterned stamp. The stamp was prepared as above, adequately cured before being placed in contact with the substrates, and treated with oxygen plasma.

The upper surfaces of the transferred pattern are not planar but uneven, because of the rupture of the bonds of the linking chains. If both surfaces were not treated, an uneven transfer was obtained, with lumps distributed irregularly, as clearly shown in
Figure 6 on the right. The same behavior was observed if only the stamp was treated with the oxygen plasma or not treated at all, and consequently, features were clearly lower.

Even though oxidation of the surface is mainly responsible for the transfer of the features, the competition between contact time and heat exposure also plays a role in determining the particular morphology of the pattern transferred. The height of the patterns increased with the contact time, because of a major possibility of 

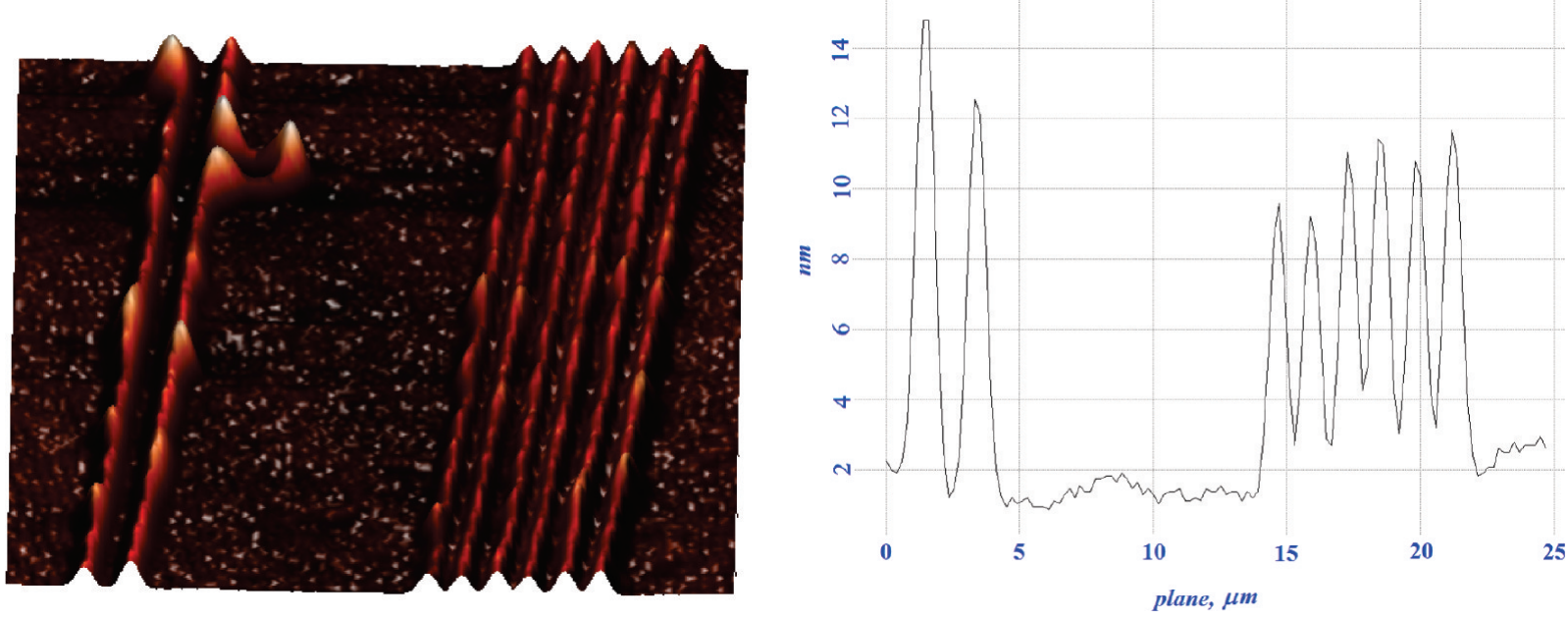

Figure 4. AFM scans of the nanopatterns obtained because of the weak bonding of the PDMS stamps to the substrate. On the right, the cross-sectional profile of the AFM image is shown.
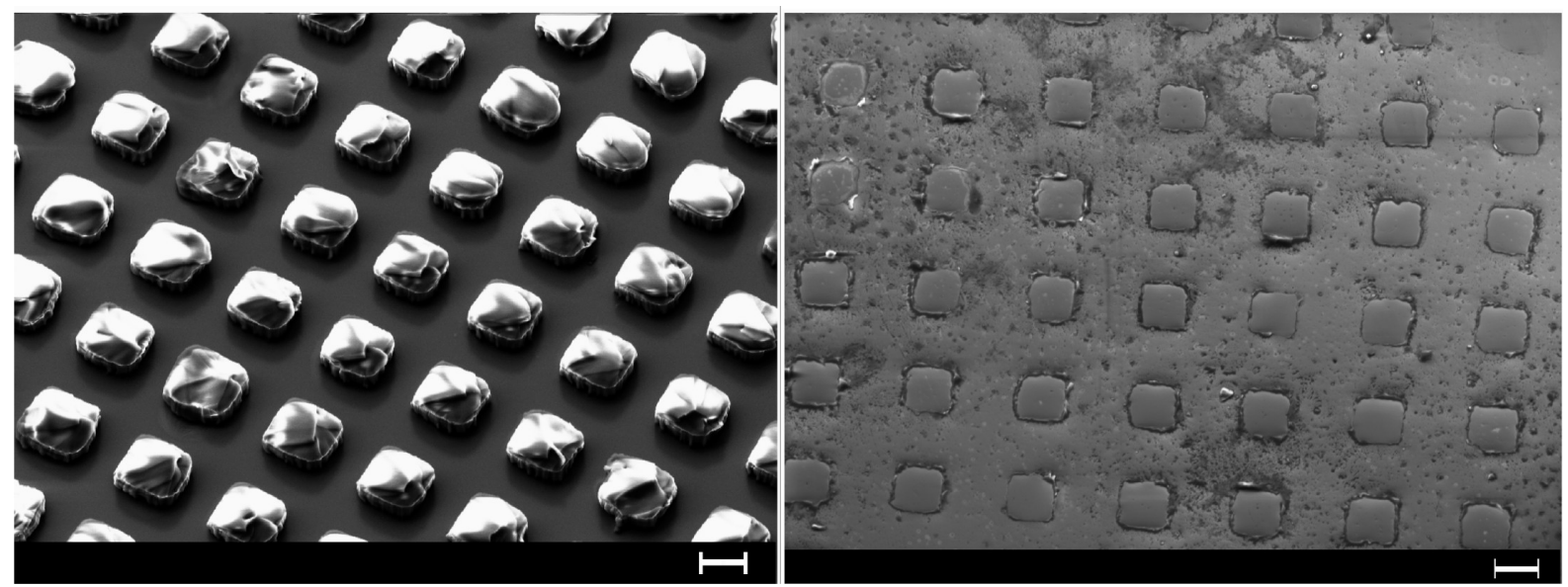

Figure 5. SEM images of the transferred PDMS features obtained using a 20:1 ratio of PDMS monomer base to curing agent. The PDMS stamps of square pillars, on the left, and holes, on the right, were easily transferred. Scale bars are $40 \mu \mathrm{m}$.
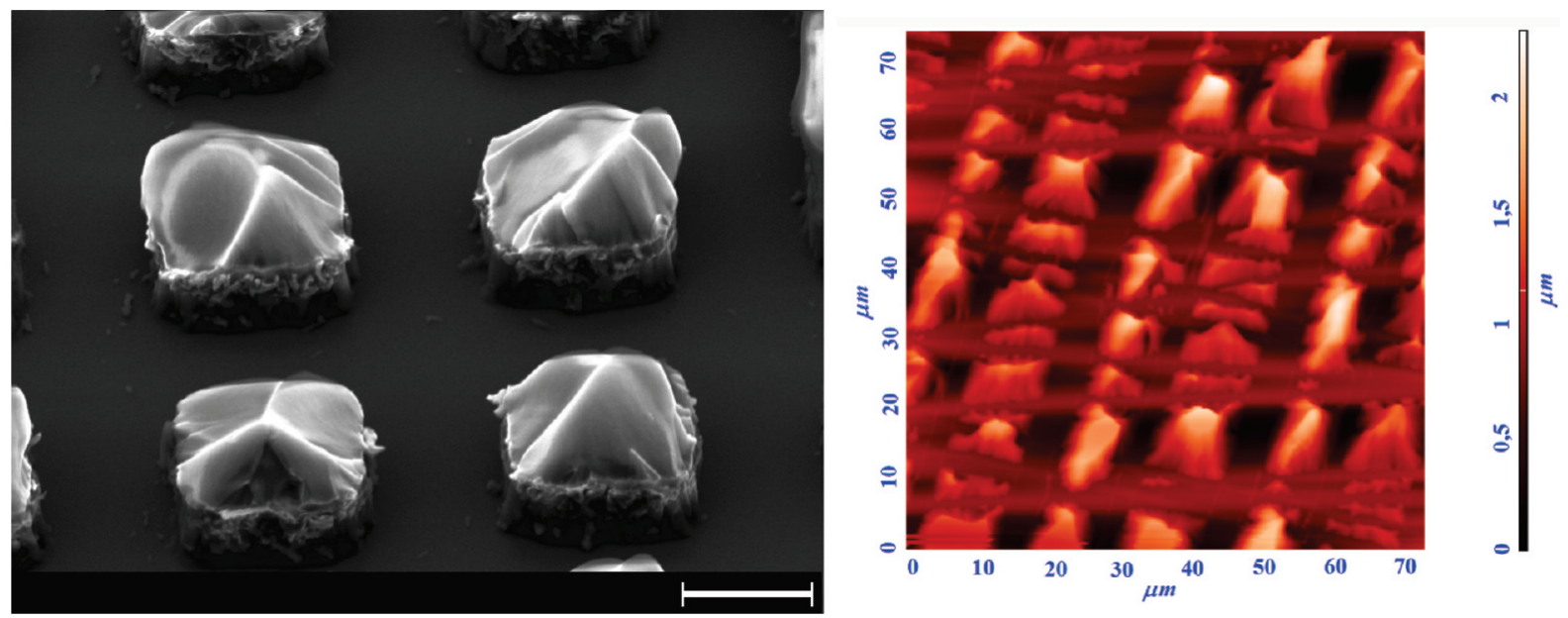

Figure 6. Ratio (20:1) of transferred PDMS squares and lines, highlighting the lack of uniformity of transferred features: on the left, a SEM image of PDMS squares, where both the stamp and substrates were plasma-treated, on the right, AFM image of PDMS lines on a substrate, where neither the stamp nor substrate was treated with the oxygen plasma and features varied ranging from 1 to $2.3 \pm 0.2 \mu \mathrm{m}$. The stamp and substrate were placed in contact for $24 \mathrm{~h}$, before peeling off the stamp. The scale bar is $40 \mu \mathrm{m}$.

diffusion of the oligomers from the bulk of the stamp to the surface. Low contact time, less than $1 \mathrm{~h}$, resulted in non-uniform residues left on the substrate because of a lack of conformal contact; therefore, transfer was obtained only in correspondence of the perimeter of the patterns. This particularly occurred in all cases, with respectively higher or lower features according as the 

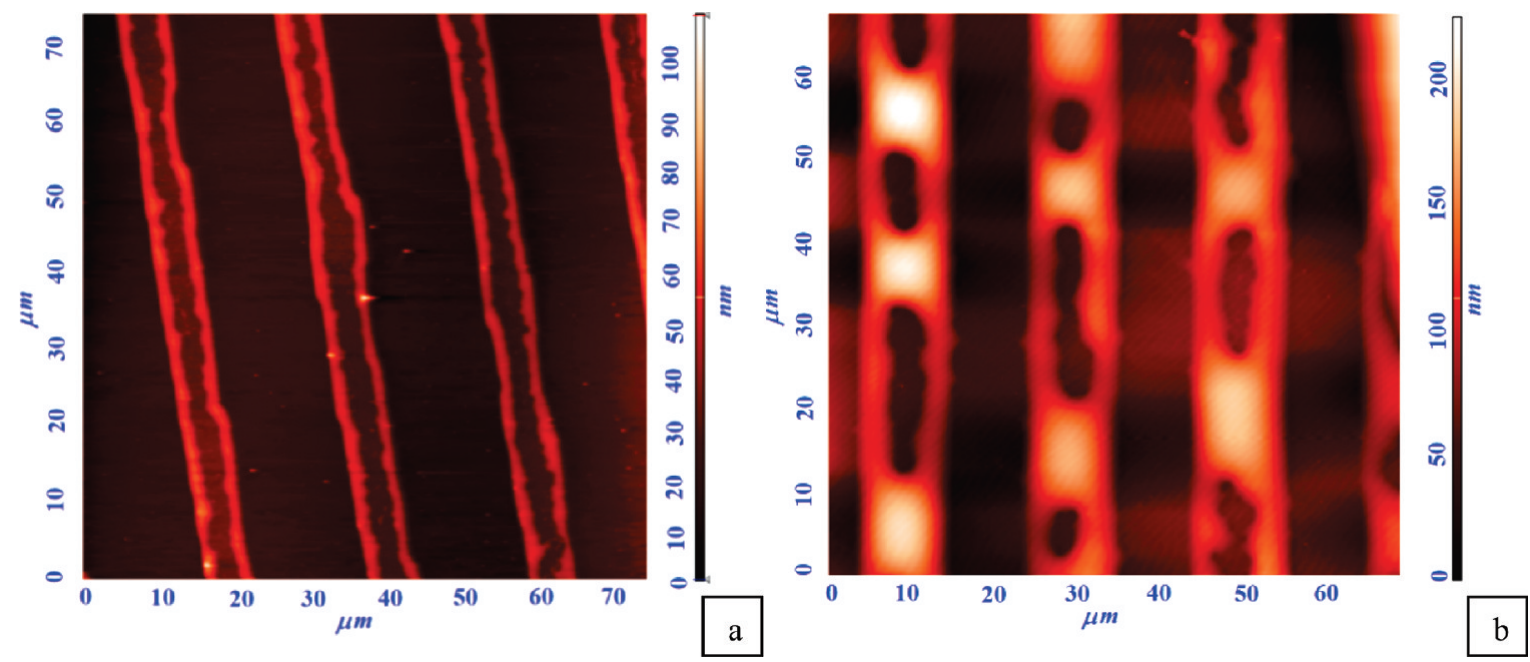

Figure 7. AFM images of transferred 10:1 PDMS lines, obtained with (a) a contact time of only half an hour and (b) excess of heat exposure, showing non-uniformity of transferred features. In both cases, only the stamp was plasma-treated, but the same occurred if both stamp and substrate were plasma-treated or not, with respectively higher and lower features.

surface was plasma-treated or not. Figure 7 shows the surface topographies obtained from 10:1 PDMS stamps with low contacting times for plasma-treated stamps. As we can see from Figure $7 \mathrm{a}$, only the rims of the lines of the stamp were transferred when the contact time was short. The height of these stripes increased with the contact time. We found that exposure to heat after conformal contact improved the bonding of the PDMS to the substrate. Whereas, it was observed that an inadequate pressure and an excess of heat speeded the transfer process but also led to an uneven transfer of the patterns to the substrate. As shown in the AFM images reported in Figure 7b, the grooves are not fully filled and it was more difficult to obtain a conformal contact with the underlying substrate.

Parameters influencing the height of the transferred features as a function of both 10:1 and 20:1 PDMS mixing ratios, plasma treatment, and contact time are summarized in Figure 8. The plot clearly shows that transferred features are related to the mixing ratio between the PDMS and the curing agent as well as the contact time between the stamp and the substrate. We note that the dimensions of features measured after $72 \mathrm{~h}$ of contact were consistently higher than those of $1 \mathrm{~h}$ contact; moreover, over $72 \mathrm{~h}$ contact features did not increase in height.

After optimizing all of the parameters, we used this method to generate more complex patterns through a multiple printing process. The pattern shown in Figure 9 was generated by a double-printing procedure: first PDMS lines were patterned onto the surface of a glass substrate with the method described above. Subsequently, the stamp was removed, and a second PDMS stamp with lines was treated with oxygen plasma and optically aligned to be placed perpendicular on top of the first set of lines. Because of the good elasticity of PDMS, a conformal contact with the patterned surfaces was obtained. Once the PDMS stamp was peeled away, the lines from the second PDMS stamp were orthogonally transferred on top of the patterned parallel lines, as illustrated by the AFM scan shown in Figure 9.

By rotating different angles between these two stamps and changing the treatment of the substrates, patterns with a variety of shapes and heights could be produced.

In comparison to the other methods proposed in the literature, the approach presented in this work shows several advantages. The key effect presented here is the formation of controlled

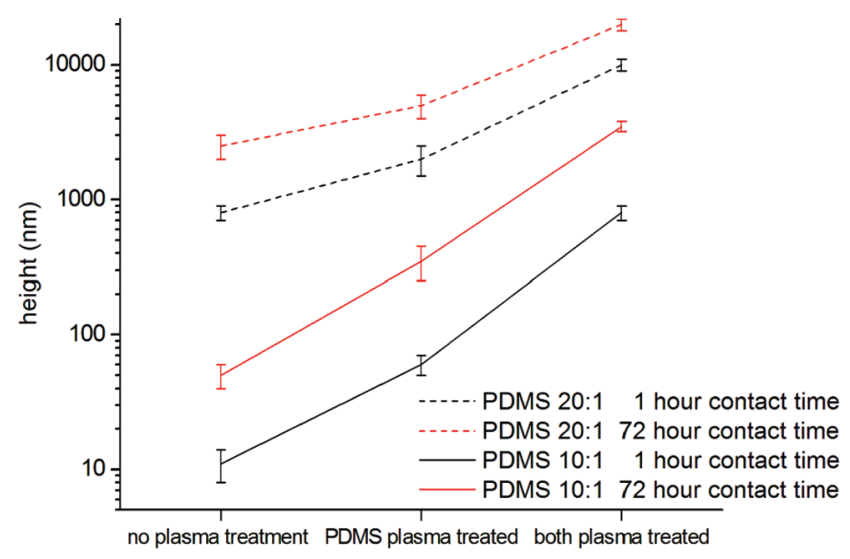

Figure 8. Plot of the height of the transferred features of the PDMS stamp as a function of the contact time, plasma treatment, and PDMS mixing ratio. Error bars represent standard error on an average of 10 experiments, all exposed in an oven at $60{ }^{\circ} \mathrm{C}$ after being placed in conformal contact. With a contact time of $1 \mathrm{~h}$, features were smaller, increasing in height as the time and PDMS ratio increased.

patterns, without the need for supplementary techniques or chemical treatments, which will lead to a more complicated process. Using different treatment parameters, our approach is expected to control the thickness of the transferred PDMS layer, allowing for a faster single-step patterning process. Depending on the treatment of the substrate and the PDMS stamp, the contact time, and the ratio of PDMS and curing agent, the feature size of transferred PDMS can be varied from $\sim 10 \mathrm{~nm}$ to $15 \mu \mathrm{m}$, without obvious differences in reproduction quality.

Such heterogeneous surfaces are useful to guide cells to spread on the wettable region and to retract from the PDMS. The PDMS features would serve in this case as a physical barrier, limiting the movement of cells. After transfer of PDMS features, the stamp was peeled off, revealing a nonfouling background, which is cellresistant. Fibroblasts h-TERT were seeded onto the micropatterned substrate and allowed to adhere for $24 \mathrm{~h}$ under standard culture conditions. Cell viability and morphological characteristics were investigated using optical microscopy and immunostaining, to obtain qualitative and quantitative information on cell alignment. 

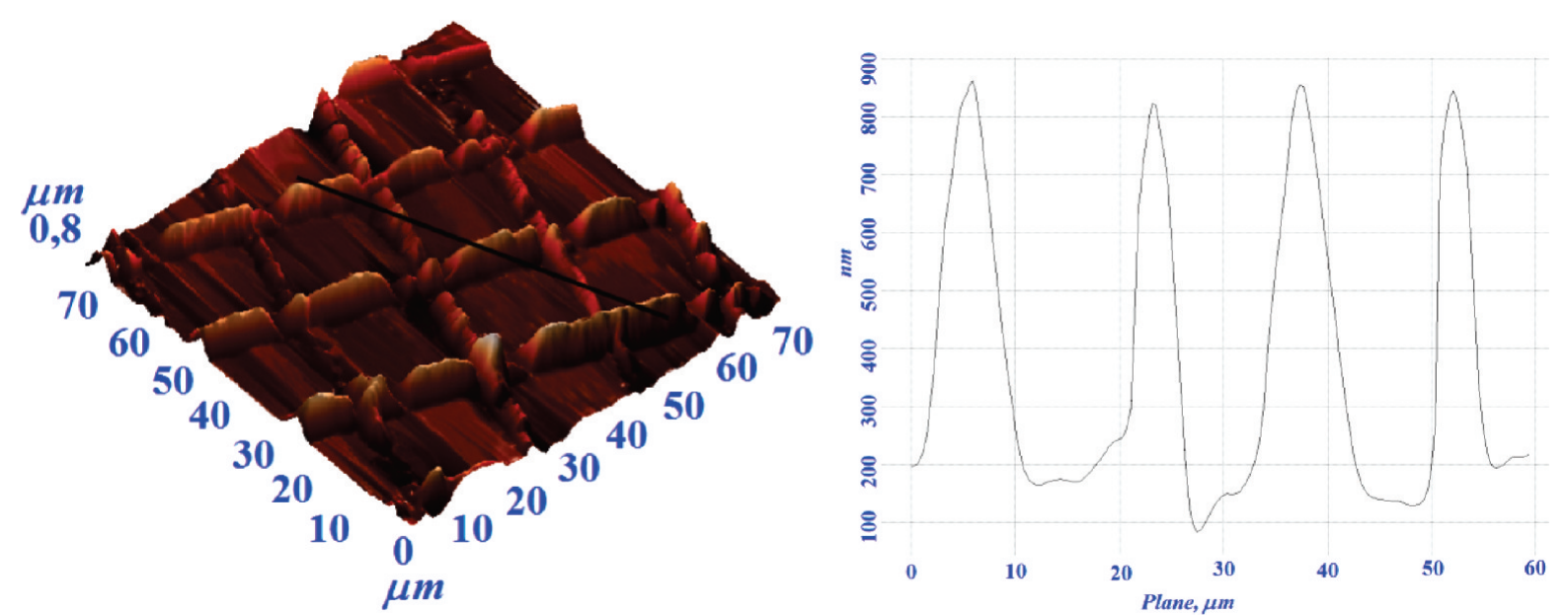

Figure 9. AFM scan with cross-sectional profile, of the multiprinting patterns obtained with a PDMS stamp with lines rotated $90^{\circ}$ on a substrate with the existing lines.

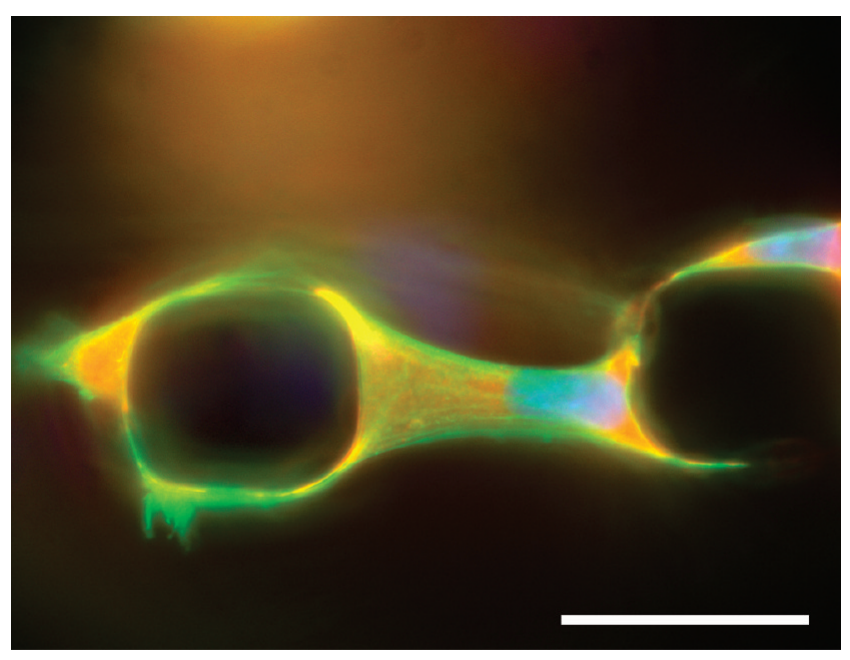

Figure 10. Immunofluoresence images of cells on patterned substrates. Cells showed a preference to the substrate avoiding the transferred patterns. The scale bar represents $50 \mu \mathrm{m}$.

As expected, cells interacted with the topography and aligned strictly only on the exposed glass substrate and not on the PDMS transferred features. Clearly, the transfer of the PDMS features approach used for the substrate shown in Figure 10 results in a highly selective and uniform cell pattern.

\section{Conclusion}

Transfer of PDMS residues from a PDMS stamp has been shown to be a rapid and reliable method to pattern surfaces of interest modifying the surface energy of a substrate. In summary, we have proposed a simple method to micro- and nanopattern surfaces using PDMS, based on oxygen activation of the surface. PDMS features of nanometric size were transferred to the substrate if neither the stamp nor substrate was plasma-treated. When the treatment of both the substrates and the master is varied systematically, the height of the surface patterning can be finely tuned and surfaces with micro- or nanotopography can be produced. Hence, specific heights are achieved depending upon the treatment of the surface and the period of contact. The proposed mechanism is that the oxygen plasma increases the number of $\mathrm{Si}-\mathrm{O}$ bonds on the stamp surface. The presence of covalent bonds enhances the strength of the interfacial adhesion between the stamp and the substrate. Thus, the proposed technique makes possible to control, uniform, and precise pattern fabrication with different heights and shapes on a large area. Here, a top-down photolithography approach is used to guide cell adhesion, integrating PDMS transfer with cell micropatterning. In this technique, the topography can be fine-tuned accord ing to the cell type to improve cellular adhesion and function. We demonstrated the effects of topography on cell adhesion. 